\title{
Elongational dynamics of multiarm polystyrene
}

\author{
Rasmussen, Henrik K.; Skov, Anne Ladegaard; Nielsen, Jens Kromann; Laille, Phillippe
}

Published in:

Journal of Rheology

Link to article, DOI:

$10.1122 / 1.3059575$

Publication date:

2009

Document Version

Publisher's PDF, also known as Version of record

Link back to DTU Orbit

Citation (APA):

Rasmussen, H. K., Skov, A. L., Nielsen, J. K., \& Laille, P. (2009). Elongational dynamics of multiarm polystyrene. Journal of Rheology, 53(2), 401-415. https://doi.org/10.1122/1.3059575

\section{General rights}

Copyright and moral rights for the publications made accessible in the public portal are retained by the authors and/or other copyright owners and it is a condition of accessing publications that users recognise and abide by the legal requirements associated with these rights.

- Users may download and print one copy of any publication from the public portal for the purpose of private study or research.

- You may not further distribute the material or use it for any profit-making activity or commercial gain

- You may freely distribute the URL identifying the publication in the public portal

If you believe that this document breaches copyright please contact us providing details, and we will remove access to the work immediately and investigate your claim 


\title{
Elongational dynamics of multiarm polystyrene
}

\author{
Henrik Koblitz Rasmussen ${ }^{\text {a) }}$ \\ Department of Mechanical Engineering, The Danish Polymer Centre, \\ Technical University of Denmark, DK-2800 Kgs. Lyngby, Denmark
}

Anne Ladegard Skov and Jens Kromann Nielsen

Department of Chemical and Biomechanical Engineering, The Danish Polymer Centre, Technical University of Denmark, DK-2800 Kgs. Lyngby, Denmark

\author{
Philippe Laillé \\ Department of Mechanical Engineering, The Danish Polymer Centre, \\ Technical University of Denmark, DK-2800 Kgs. Lyngby, Denmark
}

(Received 24 June 2008; final revision received 20 November 2008)

\begin{abstract}
Synopsis
The startup of uni-axial elongational flow followed by stress relaxation and reversed bi-axial flow has been measured for a branched polystyrene melt with narrow molar mass distribution using the filament stretching rheometer. The branched polystyrene melt was a multiarm $A_{\mathrm{q}}-C-C-A_{\mathrm{q}}$ pom-pom polystyrene with an estimated average number of arms of $q=2.5$. The molar mass of each arm is about $28 \mathrm{~kg} /$ mole with an overall molar mass of $M_{\mathrm{w}}=280 \mathrm{~kg} / \mathrm{mole}$. An integral molecular stress function constitutive formulation within the "interchain pressure" concept agrees reasonably well with the experiments.
\end{abstract}

(C) 2009 The Society of Rheology. [DOI: 10.1122/1.3059575]

\section{INTRODUCTION}

The understanding of nonlinear flow properties of polymer melts requires reliable experimental stress-strain measurements on well-characterized polymers. Considering elongational flow, one has to keep in mind that interest in the measurement of elongational viscosity of polymer melts started more than 40 years ago [Tanner and Walters (1998)], and an immense amount of scientific papers on this subject have appeared. Measurements were most frequently reported as either startup or steady elongational viscosity. In extensional rheometry for polymer melts the commonly used equipments were and still are the Münstedt tensile rheometer [Münstedt (1979)], the rheometric scientific RME [initially constructed by Meissner and Hostettler (1994)], as well as more recently presented equipments as the filament strecth rheometer (FSR) [Bach et al. (2003b)] and the Sentmanat extensional rheometer (SER) [Sentmanat (2004)].

\footnotetext{
${ }^{a)}$ Author to whom correspondence should be addressed; electronic mail: hkr@polymers.dk
} 
Especially well-defined narrow molar mass distribution (NMMD) polymer melts contribute to the understanding of the complex flow physics of polymer melts, as reptationbased [de Gennes (1971)] constitutive theories are based on the monodisperse polymer as the model molecule [Doi and Edwards $(1978,1979)$ ]. Experimental efforts on both linear and branched NMMD polymer melts have included the characterization of in startup of uni-axial extension [McLeish et al. (1999); Luap et al. (2005)] as well as steady flow following startup of uni-axial extension [Bach et al. (2003a); Nielsen et al. (2006a, 2006b)].

Recently new techniques have been presented to measure stress relaxation after extension based on the RME and SER. The appearance of a progressive thinning of the sample ending with a ductile necking limits the applicability of these techniques at high strains [Wang et al. (2007)]. The actual physical course of this necking is currently unknown, although an experimental method to measure stress relaxation omitting the necking instability has been published [Nielsen et al. (2008)]. Experimental methods to measure elongational flow more dynamically have appeared very recently. It concerns bi-axial reversed flow following uni-axial extensional flow [Nielsen and Rasmussen (2008)], as well as large amplitude oscillatory elongation [Rasmussen et al. (2008)]. Nielsen (2008); Nielsen and Rasmussen (2008); and Rasmussen et al. (2008) all use the FSR and presented dynamical extension data for a NMMD linear polystyrene melt.

An immense variety of concepts for reptation-based models exist for linear NMMD polymer melts. It was initiated by Doi and Edwards (1978, 1979) and followed by Marrucci and Grizzutti (1988); Pearson et al. (1989); Mead et al. (1998); Fang et al. (2000); Ianniruberto and Marrucci (2001); Schieber et al. (2003); Likhtman (2005); etc. Currently the only model effectively capable of predicting the published elongational flow data for linear NMMD polymer melts is the integral molecular stress function formulation [Wagner (2005)] based on the "interchain pressure" (IP) concept by Marrucci and Ianniruberto (2004) [Wagner et al. (2008); Nielsen et al. (2008); Nielsen and Rasmussen (2008); Rasmussen et al. (2008)].

For branched polymer melts the first insight into the non-linear extensional rheology of broadly-distributed polymers was obtained from the observation of time and strain separability [Wagner (1978)]. Recently, Nielsen et al. (2006a) found agreement with separability in time and strain in both the startup and steady viscosity for a NMMD branched polystyrene melt. Nielsen et al. (2006a) considered a multiarm $A_{\mathrm{q}}-C-C-A_{\mathrm{q}}$ homopolymer, often referred to as a "pom-pom" polymer. A pom-pom polymer is an important molecular architecture as it has two branch points connected by a polymer crossbar $(C)$ and a number $(q)$ of identical arms $(A)$ emanating from the branch-points. The H-polymer $(q=2)$ is the most simple type of a branched polymer. The separability was only valid at Deborah numbers for the cross-bar relaxation larger than unity and Deborah numbers for arm relaxation less than unity. The experimental observed separability is in contradiction to the H-polymer or pom-pom theory [McLeish and Larson (1998); McLeish et al. (1999)], although in shear flow, qualitative agreement is generally observed between pom-pom or H-polymer theory and experiments in both the linear viscoelastic and nonlinear shear regime [McLeish et al. (1999); Archer and Juliani (2004)].

To gain further insight into the flow physics of polymer melts, we will present direct measurements of the stress relaxation and the bi-axial reversed flow, both following (potentially steady) uni-axial extensional flow using the NMMD pom-pom polystyrene synthesized by Nielsen et al. (2006a). 


\section{ELONGATIONAL METHODOLOGY}

Our elongational experiments are performed using the currently only existing filament stretching rheometer (FSR) surrounded by a thermostatted environment, developed by Bach et al. (2003b). This rheometer allows measurements on polymeric fluids from room temperatures to $200{ }^{\circ} \mathrm{C}$. The first filament stretching rheometer (FSR) was developed by Sridhar et al. (1991). In the FSR, a cylindrical shaped liquid sample, with height $L_{\mathrm{i}}$ and radius $R_{\mathrm{i}}$, is placed between two parallel solid cylinders having the same diameter as the pellet. Separation of the plates will extend the sample. The FSR measures the strain in the sample at exactly the location of the necking or the midfilament plane of the extended sample. Subsequently, this critical region can be monitored using laser microscopy and the distance between the end-plates adjusted, thus obtaining a predefined stretch rate at the neck. To ensure a correct measurement the sample should stay symmetric across the mid-filament plane as well as axisymmetric during extension. Loss of symmetry due to gravitational sagging of the filament can be neglected for almost all polymer melts. However, one has to keep in mind that the rheological behavior of the polymer may in itself result in symmetry breaking [Matallah et al. (2006)] as well as axis-symmetric breaking [Rasmussen and Hassager (2001)] phenomena.

The original FSR configuration by Bach (2003b) maintains a constant extension rate. Through a feedback mechanism, the separation of the end plates is controlled and the elongational rate is thereby kept constant. Modification of this closed loop control mechanism has enabled measurements of stress relaxation [Nielsen (2008)] and bi-axial reversed flow [Nielsen and Rasmussen (2008)], both following uni-axial extensional flow as well as large amplitude oscillatory elongation (LAOE) [Rasmussen et al. (2008)]. These experimental techniques to measure dynamically complex elongational flow on polymer melts with a well-defined and controlled deformation rate are, according to our knowledge, the only ones published. The method by Nielsen et al. (2008) prevents the commonly observed necking instability during stress relaxation [Wang et al. (2007)]. The method by Nielsen and Rasmussen (2008) monitors a well-defined reversed flow, where the flow during elastic recovery [Meissner (1971)] is unknown. The elastic recovery is measured as the maximal recoverable Hencky strain in the reversed bi-axial flow following uni-axial elongation flow. It is measured without externally applied forces on the sample during the reversed flow.

During the extension in a FSR, a load cell measures the elongational force, $F(t)$, and a laser micrometer measures the filament diameter, $2 R(t)$, at the mid-filament plane. The relevant strain in the elongation is the Hencky strain $(\epsilon)$, which is defined as $\epsilon(t)$ $=2 \ln \left(R_{0} / R(t)\right)$ for filament stretching of cylindrically shaped samples. $R_{0}$ is the midfilament radius of the sample (and $L_{0}$ the length) at the start of the extension, at time $t$ $=0$, as the sample may have been subjected to a pre-stretch. Initially, the sample is at rest for times $t<0$. The elongational or stretch rate is defined as $\dot{\epsilon}=d \epsilon / d t$.

The elongational stress generated within the sample filament can be calculated from the total force measured by the load cell as [Szabo (1997)]

$$
\sigma_{\mathrm{zz}}-\sigma_{\mathrm{rr}}=\frac{F(t)+\rho \pi R_{\mathrm{i}}^{2} L_{\mathrm{i}} g / 2}{\pi R(t)^{2}}-\frac{\sigma}{R(t)},
$$

where $\sigma$ is the surface tension, $\rho$ the density of the polymer melt, and $g$ the gravitational acceleration. Eq. (1) is written without taking the correction for the fluid inertia into account. If the gravitational sagging is unimportant, which is true for almost all polymer melt measurements, the inertial term is negligibly small. A thorough discussion on the fluid inertia correction can be found in Szabo and McKinley (2003). 
One of the characteristics of elongational flow in a filament stretching apparatus is that the extension is not uniform in the direction of the extension of the fluid as a result of the no-slip at the end plates. The force balance from Szabo (1997) denotes an average of the extensional stress over the mid-filament plane. The FSR relies on the assumption of a homogeneous elongation in the mid-filament plane as the kinematics is measured at this center of the sample. Numerical simulation of the filament stretch in axis-symmetry as well as fully three dimensional (3D) has been published during the last decade. They include papers by Kolte et al. (1997); Sizaire and Legat (1997); Hassager et al. (1998); Yao et al. (1998); Yao et al. (2000); Sujatha et al. (2006); Matallah et al. (2007), and Webster et al. (2008) in axis-symmetry and Rasmussen and Hassager (1999) and Bach et al. (2002) in fully 3D. These simulations have considered a wide variety of constitutive models and they all confirmed that deviations from ideal elongational flow, especially at higher Hencky strain, are small near the mid-filament plane.

At small aspect ratios $A_{\mathrm{i}}=L_{\mathrm{i}} / R_{\mathrm{i}}$, an extra shear contribution may add to the measured elongational force during the startup of the flow. The effect of the additional shear may be reduced by a correction factor [Spiegelberg et al. (1996)] as

$$
\left(\sigma_{\mathrm{zz}}-\sigma_{\mathrm{rr}}\right)_{\mathrm{corr}}=\frac{\left(\sigma_{\mathrm{zz}}-\sigma_{\mathrm{rr}}\right)}{1+\frac{\left(R(t) / R_{\mathrm{i}}\right)^{14 / 3}}{3 A_{\mathrm{i}}^{2}}}
$$

to a level where the true elongational stress usually stays within the experimental scattering of the corrected stress. The correction factor is less accurate at increasing strains, where the effect of the correction factor fortunately vanishes [Kolte et al. (1997)]. This corrected transient uniaxial elongational stress is defined in a potentially pre-stretched configuration. The pre-stretch, performed prior to the elongational experiments, avoids an initial large correction. Notice that the correction is negligible (less than 10 percent) after less than 0.7 strain units of $\epsilon$ in the the startup of flow, and without importance during both the stress relaxation and the reversed bi-axial flow. The use of Eq. (2) requires an extension rate during pre-stretch considerably lower than the inverse of the largest relaxation time in the melt.

In this paper we intend to perform transient uniaxial stretching up to a Hencky strain of $\epsilon_{0}$. This uniaxial stretching is followed by either transient biaxial squeeze or stress relaxation. Generally, the relative deviation between measured and expected radius never exceeded $3 \%$.

During the startup of uniaxial stretching we define the rate $\dot{\epsilon}^{+}=\dot{\epsilon}$ and in the biaxial squeeze the rate is defined as $\dot{\epsilon}^{-}=-\dot{\epsilon}$. Both $\dot{\epsilon}^{+}$and $\dot{\epsilon}^{-}$will stay positive, constant, and equal $\left(\dot{\epsilon}^{+}=\dot{\epsilon}^{-}\right)$during the extension. An important quantity in the reversing flow experiment is the Hencky strain at which the stress in the filament changes sign (at time $t_{R}$ ) from positive to negative stress. The needed bi-axial strain or "strain recovery" $\left(\epsilon_{R}\right)$ to reach zero extensional stress is $\epsilon_{R}=\epsilon_{0}-\epsilon\left(t_{R}\right)$. Here $t_{R}$ is the time where $\sigma_{\mathrm{zz}}-\sigma_{\mathrm{rr}}=0$. For further details regarding the methodology and definitions, please refer to Nielsen and Rasmussen (2008).

During the stress relaxation, starting at a Hencky strain of $\epsilon_{0}$, the mid-filament radius is kept constant $(\dot{\epsilon}=0)$. The viscosities during both startup and stress relaxation are defined as 


$$
\bar{\eta}^{+}=\frac{\sigma_{\mathrm{zz}}-\sigma_{\mathrm{rr}}}{\dot{\epsilon}^{+}} \text {and } \bar{\eta}^{-}=\frac{\sigma_{\mathrm{zz}}-\sigma_{\mathrm{rr}}}{\dot{\epsilon}^{+}}
$$

respectively. The viscosity during the stress relaxation is calculated applying the constant elongational rate $\left(\dot{\epsilon}^{+}=\dot{\epsilon}\right)$ from the preceding flow. For further experimental details we refer to Nielsen et al. (2008).

\section{POM-POM POLYSTYRENE}

The branched polystyrene melt used in this work is the same batch as synthesized by Nielsen et al. (2006a) based on the method developed by Knauss and Huang (2001): A NMMD multiarm $A_{\mathrm{q}}-C-C-A_{\mathrm{q}}$ pom-pom polystyrene with a mass average molar mass of $M_{w}=280 \mathrm{~kg} / \mathrm{mole}$ prepared by anionic polymerization. The number of arms is not a fixed value but rather a distribution given by the stoichiometry. Here with an number average value of $q$ of approximately 2.5 , whereas the arm (with a number average molar mass of $M_{\mathrm{n}, \mathrm{A}}=28 \mathrm{~kg} /$ mole $)$ and backbone length $\left(M_{\mathrm{n}, \mathrm{B}} \approx 140 \mathrm{~kg} /\right.$ mole $)$ are narrow distributed [polydispersities of $\mathrm{PDI}(\mathrm{A}) \approx \mathrm{PDI}(\mathrm{C}) \approx 1.06$ ]. They report the molar fraction or ratio of $n$-armed stars $\left(p_{n}\right)$ emanating from the branch points in the pom-pom polystyrene to be $\left(p_{2}, p_{3}, p_{4}\right)=(0.571,0.354,0.075)$ in the melt. Assuming ideal reaction kinetics we calculate the molar ratios between the different pom-pom molecules to $\left(p_{2} p_{2}, 2 p_{2} p_{3}, 2 p_{2} p_{4}, p_{3} p_{3}, 2 p_{3} p_{4}, p_{4} p_{4}\right)=(0.326,0.404,0.086,0.125,0.053,0.006)$. A full closure regarding the polymerization and characterization of molecular structure can be found in Nielsen et al. (2006a).

The pom-pom polystyrene batch or reaction mixture polymerized by Nielsen et al. (2006a) needs to be fractionated several times to remove low molecular by-products using toluene/methanol as solvent/non-solvent pair for fractional precipitation of the highest molar mass polystyrene component. Notice that in Nielsen et al. (2006a) this fraction was estimated to be about $7 \mathrm{wt} \%(6-9 \mathrm{wt} \%)$ in the pom-pom polymer used in their elongational measurements. The minor amount of by-products left in the fractioned pom-pom mixture consists of almost half pom-pom molecules, here referred to as asymmetric star molecules. In our work the sample was fractionated to a level containing approximately $20 \mathrm{wt} \%$ asymmetric stars present in the melt.

\section{STRESS RELAXATION}

Figure 1 shows the measured corrected transient elongational viscosity, $\bar{\eta}_{\text {corr }}^{+}(t)$, followed by the viscosity $\bar{\eta}^{-}(t)$ during the stress relaxation for the pom-pom polystyrene with predictions from the linear viscoelastic properties (LVE) from Nielsen et al. (2006a). The measured transient elongational viscosity from Nielsen et al. (2006a) and one new startup experiment at $\dot{\epsilon}=0.3 \mathrm{~s}^{-1}$ are shown as well. In the plot there is agreement between all measurements and the LVE predictions up to a certain level of strain. Furthermore, our measurements and the measurements published by Nielsen et al. (2006a) agree within $20 \%$.

It is of interest to compare the experimental observations with predictions from a time-strain separability model (Wagner, 1978). For this purpose we introduce the integral molecular stress function (MSF) formulation by Wagner and Schaeffer (1992). The general form of the MSF constitutive model is written as a memory-weighted time integral over a strain tensor and the square of the molecular stress function, $f^{2}$, 


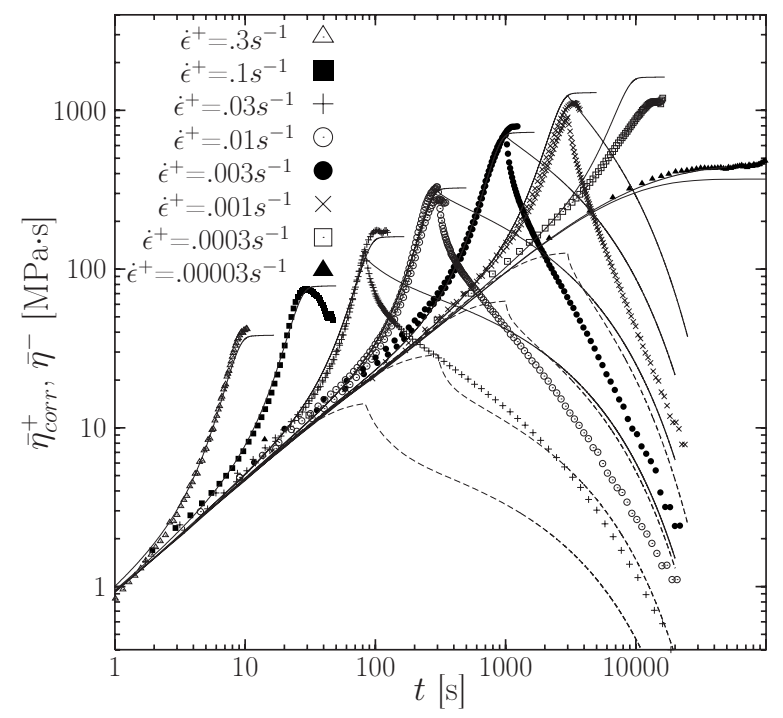

FIG. 1. The measured corrected startup and relaxation viscosity performed at rates of $\dot{\epsilon}^{+}=\dot{\epsilon}$

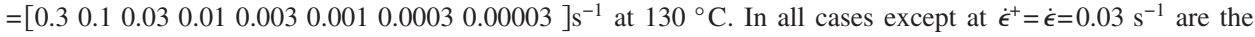
flow stopped at an extension of $\epsilon_{0}=3$ and allowed to relax. The flow is stopped at a Hencky strain of $\epsilon_{0}=2.5$ at $\dot{\epsilon}^{+}=\dot{\epsilon}=0.03 \mathrm{~s}^{-1}$. The solid lines (-) are the K-BKZ predictions of the transient elongational viscosity. The dashed lines (-----) are the Doi-Edwards predictions of the transient elongational viscosity (with $f=1$ ).

$$
\sigma_{\mathrm{ij}}=\int_{-\infty}^{t} M\left(t-t^{\prime}\right) f\left(t, t^{\prime}\right)^{2} 5\left\langle\frac{E_{\mathrm{in}} u_{\mathrm{n}} E_{\mathrm{jm}} u_{\mathrm{m}}}{|\mathbf{E} \cdot \mathbf{u}|^{2}}\right\rangle d t^{\prime} .
$$

If the molecular stress function $f$ is strain-dependent only, an applicable time-strain separable model is obtained. The terms $\sigma_{\mathrm{ij}}$ are the integral components of the stress tensor. The angular brackets denote an average over a unit sphere $\langle\cdots\rangle=1 /(4 \pi)$ $\int_{|\mathbf{u}|=1} \cdots d \mathbf{u}$ where a tube segment of unit length and orientation is given by the unit vector $\mathbf{u}$. In the stress-free state, $\mathbf{u}$ is deformed into $\mathbf{E} \cdot \mathbf{u}$ in the current state. These brackets are the Doi-Edwards strain tensor with the independent alignment approximation (IAA) [Doi and Edwards $(1978,1979)]$ and the Doi-Edwards model with the IAA is obtained for $f=1$. The components of the macroscopic displacement gradient tensor is given by $E_{\mathrm{ij}}\left(\mathbf{x}, t, t^{\prime}\right)=\partial x_{\mathrm{i}} / \partial x_{\mathrm{j}}^{\prime}, i=1,2,3$ and $j=1,2,3 .\left(x_{1}^{\prime}, x_{2}^{\prime}, x_{3}^{\prime}\right)$ is the coordinates of a given particle in the stress free reference state (time $\left.t^{\prime}\right)$, displaced to coordinates $\left(x_{1}, x_{2}, x_{3}\right)$ in the current state (time $t$ ). The memory function $M\left(t-t^{\prime}\right)$ is related to the relaxation modulus as $M\left(t-t^{\prime}\right)=d G\left(t-t^{\prime}\right) / d t^{\prime}$. The parameters in a double mode BSW (BaumgaertelSchausberger-Winter) model [Baumgaertel et al. (1990); Jackson and Winter (1995)] defining the relaxation modulus for the pom-pom melt can be found in Nielsen et al. (2006a).

The MSF constitutive model has, in general, been successful in describing two- and three-dimensional complex extensional dominated flows of linear and branched melts within the experimental error [Wagner et al. (1998); Rubio and Wagner (2000); Eriksson and Rasmussen (2005); Rasmussen and Bach (2005); Rasmussen and Eriksson (2007); Rasmussen and Yu (2008)]. These papers are mainly based on a K-BKZ [Kaye (1962); Bernstein et al. (1963)] version of the MSF model. The situation is less satisfactory for branched polymers where the presence of both shear and extension flow appears to be a more difficult task for the MSF model [Olley and Wagner (2006)]. 
Here we will initially use a simplified K-BKZ version of the MSF model to allow for the time-strain separability of the form

$$
f^{2}=1+\left(f_{\max }^{2}-1\right)\left[1-\exp \left(-\frac{\exp (2 \ln |\mathbf{E} \cdot \mathbf{u}|)-1}{f_{\max }^{2}-1}\right)\right]
$$

representing a simple elasticity strain with affine movement of cross-links [Flory (1976)], where the molecular stress function is based on the potential function from Doi-Edwards theory with the IAA. $f_{\max }$ is the maximal value of the molecular stress function and is easily obtained from Nielsen et al. (2006a), from their figure 15 as $f_{\max }^{2}=18$. Figure 1 shows the measured viscosities together with this time-strain separable K-BKZ model.

The time-strain separability agrees quantitatively with the start-up and steady elongational viscosities $\bar{\eta}^{+}$, within the limit mentioned in the Introduction section as expected. However, it fails completely in the stress relaxation. The time-strain separability predicts stresses up to a factor $10(1000 \%)$ larger then the experimental observed during the stress relaxation. A time-strain strain separable model does not include the possibility of time relaxation in the strain, physically interpreted as relaxation in the stretch of the molecule.

In Fig. 1, the predictions of the model with $f=1$ (equivalent to $f_{\max } \rightarrow 1$ ) are also shown. It corresponds to the strain from the Doi-Edwards reptation theory which is generally accepted to be lower limit of the strain function. For linear NMMD melts the stress converges toward a Doi-Edwards strain based model at large times. This has recently been experimentally documented for elongation of linear NMMD polystyrene [Nielsen and Rasmussen (2008)]. In fact, the stress in the pom-pom melt seems to converge toward a Doi-Edwards strain-based model at large times similar to linear melts. At steady flow the measured stresses are ten times higher than the Doi-Edwards stress, where the deviation between a Doi-Edwards strain based model and the experiments are less than a factor of 2 at the end of the experiments. Notice that the experiments cannot be followed further in time due to the resolution on the weight cell. The weight cell measures the extensional force.

Wagner et al. (2005) and Rolón-Garrido et al. (2006) adopted the "interchain pressure" concept within the MSF model for the extensional behavior in monodisperse linear polystyrene melts. It allows relaxation in the stretch of the molecule. To account for the extensional behavior in NMMD branched melts we will do the same. Inspired by their work, we use an integral molecular stress function (MSF) formulation as

$$
\frac{\partial}{\partial t} f=f\left[\frac{\partial}{\partial t}\langle\ln |\mathbf{E} \cdot \mathbf{u}|\rangle-\frac{1}{\tau_{\mathrm{dia}}\left(t-t^{\prime}\right)} c\left(f, t-t^{\prime}\right) f \cdot\left[\left\{c\left(f, t-t^{\prime}\right) \cdot f\right\}^{3}-1\right]\right] .
$$

Note that the dependence of $t$ and $t^{\prime}$ in the notation on $f$ is dropped, although $f$ is strictly a function of both $t$ and $t^{\prime}$ as $f\left(t, t^{\prime}\right)$ with initial condition $f\left(t^{\prime}, t^{\prime}\right)=1 . \tau_{\text {dia }}$ is referred to as the tube diameter relaxation time. $c$ is a nonlinear spring coefficient represented by a relative Pade approximation [Cohen (1991)]. We have indicated a time dependency in both the nonlinear spring coefficient $c$ and the tube diameter relaxation time $\tau_{\text {dia }}$ in order to account for the slight polydispersity in a simplified way as discussed in the Discussion section. Marrucci and Ianniruberto (2004) and Wagner et al. (2005) did not include finite extensibility and, therefore, assumed $c=1$, whereas Rolón-Garrido et al. (2006) introduced the use of finite extensibility using relative Pade inverse Langevin function [Ye and Sridhar (2005)] 


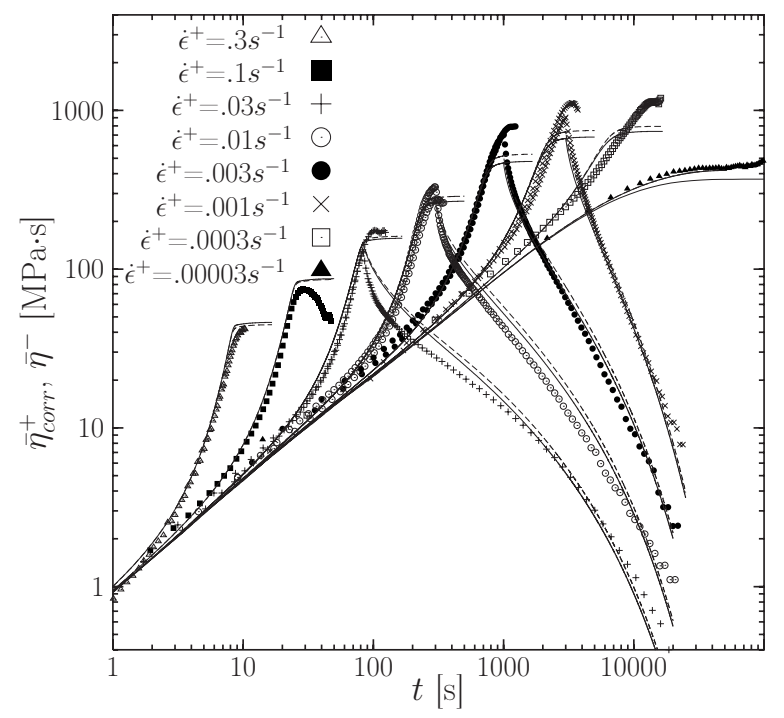

FIG. 2. The data from Fig. 1 compared to different theoretical models. The solid lines are the ideal MSF model in Eq. (6) with constant (time independent) tube diameter relaxation time $\left(\tau_{\text {dia }}\right)$ and nonlinear spring coefficient (c). A tube diameter relaxation time of $\tau_{\mathrm{dia}}=150000 \mathrm{~s}$ and a $\lambda_{\max }=5.3$ are applied. The dashed lines (-----) are the MSF model including the dilution effect from the presence of the "asymmetric stars" in the polystyrene melt.

$$
c(\lambda)=\frac{\left(3-\lambda^{2} / \lambda_{\max }^{2}\right)\left(1-1 / \lambda_{\max }^{2}\right)}{\left(3-1 / \lambda_{\max }^{2}\right)\left(1-\lambda^{2} / \lambda_{\max }^{2}\right)},
$$

where $\lambda_{\max }$ is the stretch of the fully-extended molecule. We use the same approximation in our work. Please notice that we have introduced finite extensibility on all stretch terms in the evaluation of the interchain pressure, were Rolon-Garrido et al. (2006) only applied it on the $f$ term outside the \{\} in Eq. (6). An estimate of the extension at which the molecule on average becomes fully extended is needed. For polystyrene the maximal tube length $\lambda_{\max }=5.07$ according to Rolón-Garrido et al. (2006) for the pure polystyrene. This value is based on Fang et al. (2000).

The presence of 7 or $20 \mathrm{wt} \%$ asymmetric stars (half pom-pom's) in the melt give rise to a slight dilution effect on the maximal extensibility of $\lambda_{\max } \approx 5.07 / \sqrt{1-w}$ and on the tube-diameter relaxation time of $\tau_{d i a} \approx \tau_{d i a, 0} /(1-w)^{2}$ for times above a characteristic relaxation time of the asymmetric stars polystyrene in the melt [Wagner (2008)]. Here we have used an average value of $\lambda_{\max }=5.3$ to account for the slight dilution of the polymer due to the presence of the asymmetric star molecules in the melt.

It appears from Fig. 2 that the stress relaxation data are well described, within about $30 \%$, by the interchain pressure model in Eq. (6), using a fitted (and average) tube diameter relaxation time of $\tau_{\mathrm{dia}}=150000 \mathrm{~s}$. The interchain pressure concept captures the stress relaxation in the pom-pom polystyrene, in fact slightly more accurate than previously shown for linear melts [Nielsen et al. (2008)].

\section{REVERSED EXTENSIONAL FLOW}

In Fig. 3 we show the measured recovery strain as a function of the elongational rate. The recovery strain $\epsilon_{R}$ increases with $\dot{\epsilon}$ and reaches a saturation of the values at high strain rates. The melt becomes less elastic as it gradually moves from the backbone 


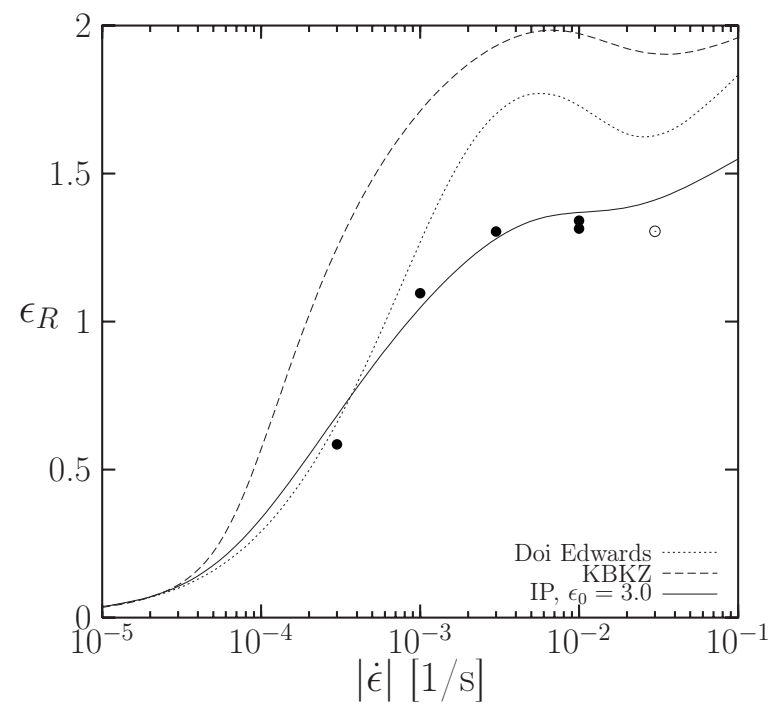

FIG. 3. Recovery strain value, $\epsilon_{R}$, plotted against elongational rate (at $130{ }^{\circ} \mathrm{C}$ ). The flow is reversed at an extension of a Hencky strain of $\epsilon_{0}=3$ with only one exception. The circle $(O)$ is one measurement where the flow is reversed at an extension of $\epsilon_{0}=2.5(\bigcirc) . \dot{\epsilon}^{+}=\dot{\epsilon}^{-}=|\dot{\epsilon}|$ in all experiments. The dashed line (-----) is the K-BKZ predictions of the experimental data. The dotted line $(\cdots \cdots)$ is the Doi-Edwards model equation $(f$ $=1$ ) representing all possible stretch evolution equation. The solid line $(-)$ is the MSF model Eq. (6) with a tube diameter relaxation time of $\tau_{\mathrm{dia}}=150000 \mathrm{~s}$ and a $\lambda_{\max }=5.3$. Hence with constant (time independent) tube diameter relaxation time $\left(\tau_{\mathrm{dia}}\right)$ and nonlinear spring coefficient $(c)$. The bullets $(\boldsymbol{)})$ are measurements where the flow is reversed at an extension of a Hencky strain of $\epsilon_{0}=3(0)$

dynamics into a region also characterized by the arm dynamics. The arm Deborah number gradually increases toward unity at the highest strain rate [Nielsen et al. (2006a)].

In Fig. 3 we have also shown the prediction for the strain recovery $\epsilon_{R}$ found using the MSF model Eq. (4) with both the time-strain separable K-BKZ model and the interchain pressure assumption. The interchain pressure concept captures the reversed flow behavior as previously shown for linear melts [Nielsen and Rasmussen (2008)]. We have also included the prediction for the Doi-Edwards model $[f=1$ in Eq. (4)]. A molecular stress function formulation considers the stretch for all deformed segments, whereas the DoiEdwards model operates with an averaged orientation factor [Doi and Edwards (1986)]; a stretch evolution equation. As shown in Nielsen and Rasmussen (2008), the strain recovery is $\epsilon_{R}$-independent of the choice of stretch evolution equation. Neither the K-BKZ nor the Doi-Edwards model with any stretch evolution function are able to predict the level of the recovery strain. As a more fundamental problem with both the K-BKZ and Doi-Edwards model, the recovery strain $\left(\epsilon_{R}\right)$ continues to increase with increasing $\epsilon_{0}$ value for $\epsilon_{0} \geqslant 3$, where steady state have been reached, i.e., the mentioned models do not capture the concept of steady state in reversing flows.

The measured extensional stress as a function of the Hencky strain for the reversing flow experiments are shown in Fig. 4. It shows the transient startup followed by the reversing flow. Analyzing the observed stress in the reversed flow with the theoretical MSF approach within the interchain pressure concept we find that the predictions from the MSF model are somewhat larger than the experimental values. However, we find agreement between the experimental data and the interchain pressure concept. 


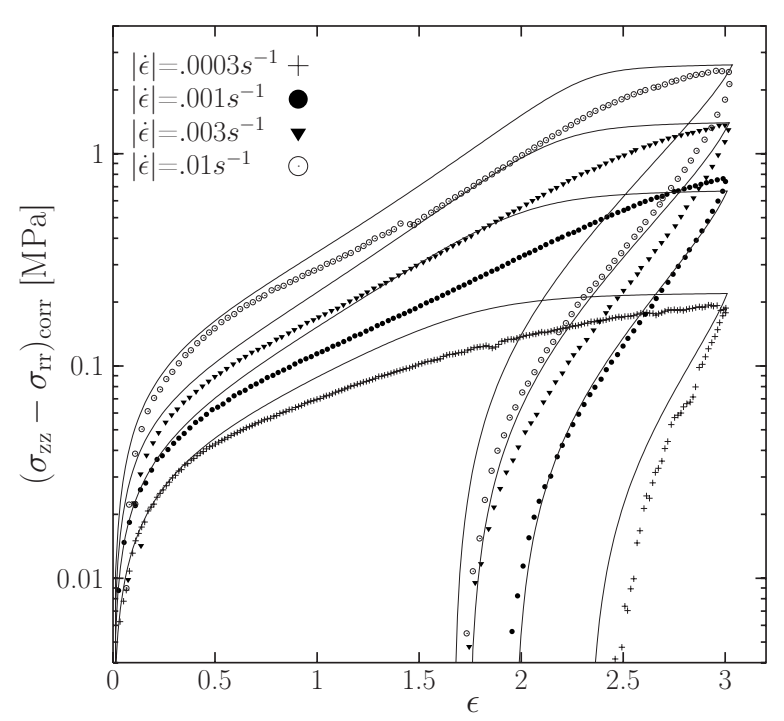

FIG. 4. Startup and reversing elongational experiments with $\dot{\boldsymbol{\epsilon}}^{+}=\dot{\boldsymbol{\epsilon}}^{-}=|\dot{\boldsymbol{\epsilon}}|$. The measurements are done for the following elongational rates: $\dot{\epsilon}^{+}=\dot{\epsilon}^{-}=0.0003 \mathrm{~s}^{-1}, 0.001 \mathrm{~s}^{-1}, 0.003 \mathrm{~s}^{-1}$ and $0.01 \mathrm{~s}^{-1}$ (at $130{ }^{\circ} \mathrm{C}$ ). In all cases the flows are reversed at an extension of $\epsilon_{0}=3$. The lines (-) are the MSF model corresponding to the experiments; Eq. (6) with constant (time-independent) tube diameter relaxation time of $\tau_{\mathrm{dia}}=150000 \mathrm{~s}$ and $\lambda_{\max }=5.3$.

\section{DISCUSSION}

The pom-pom polystyrene melt used in this work is not idealy monodisperse. The molar mass of the backbone and arms are narrow distributed, whereas two $(q=2)$, three $(q=3)$, or $(q=4)$ arms connect to the branchpoints. Please notice we use the notation $\mathrm{p}$, $\mathrm{p} 3$, and $\mathrm{p} 4$ for a number of arms connected to a branch point of $q=2, q=3$, and $q=4$, respectively.

The distribution of the different fractions of the pom-pom melt can be calculated from combinational statistics based on the spectroscopic data. Notice that the fraction of p4-p4 is considered vanishing. The relaxation time of the crossbar material will depend on the number of arms in both ends since the following equation holds for monodisperse pompoms (both in arm number and molecular weight) [McLeish and Larsen (1998)]:

$$
\tau_{\mathrm{b}}=\frac{4}{\pi^{2}} s_{\mathrm{b}}^{2} \phi_{\mathrm{b}} \tau_{\mathrm{a}}(0) q
$$

where $s_{b}=M_{b} / M_{e}$ is the crossbar length, $\phi_{b}$ the fraction of crossbar material, and the longest relaxation time of the arm material is given by

$$
\tau_{\mathrm{a}}(0)=\tau_{0} \exp \left[\frac{15}{8} s_{\mathrm{a}}-\left(1-\phi_{\mathrm{b}}\right) \frac{1}{3}\right],
$$

where $s_{a}=M_{a} / M_{e}$ is the arm length and $\tau_{0}$ is the Rouse relaxation time. Since the amount of the p4-p4 pom-pom is vanishing, we then proceed to calculate the amounts of the two different types of crossbars, namely the ones consisting of one or more p2 units (denoted q2) and the ones consisting of one or more p3 or p4 units (but no p2 units), which are denoted by $\mathrm{q} 3$. We get $\left(q_{2}, q_{3}\right)=\left(p_{2} p_{2}+2 p_{2} p_{3}+2 p_{2} p_{4}, p_{3} p_{3}+2 p_{3} p_{4}\right)=(0.82,0.18)$ by mole, which by weight fraction of the total melt converts into $\left(\mathrm{ww}\left(q_{2}\right), \mathrm{ww}\left(q_{3}\right)\right)$ $=(0.35,0.08)$. We characterize the crossbars this way in order to account for polydisper- 
sity in a very simplified way. We assume that the relaxation of the two types of crossbar is hierarchical and that a (p2-p2) and a (p2-p3) crossbar will relax at the same timescale since the arms on the p2 end will determine the time scale at which the crossbar can retract. Hence, we can, as an approximation, write the fraction of unrelaxed material as

$$
\phi(t)=\phi_{\mathrm{a}} \int_{0}^{1} \exp \left(-t / \tau_{\mathrm{a}}(x)\right) \mathrm{dx}+\left(\phi_{\mathrm{q} 2}+\phi_{\mathrm{q} 3}\right) \exp \left(-t / \tau_{\mathrm{b} 2}\right)+\phi_{\mathrm{q} 3} \exp \left(-t / \tau_{\mathrm{b} 3}\right)
$$

where $\tau_{\mathrm{b} 2}$ and $\tau_{\mathrm{b} 3}$ are calculated from Eq. (8) with $q=2$ and $q=3$, respectively.

The molecular entanglement weight $M_{\mathrm{e}}=13500 \mathrm{~g} \mathrm{~mol}^{-1}$ at $413 \mathrm{~K}$ is taken from Fetters et al. (1994) and the Rouse relaxation time $\tau_{0}=0.6 \mathrm{~s}$ at $130{ }^{\circ} \mathrm{C}$ is determined from the LVE data of Nielsen et al. (2006b). It is obvious that the effect of polydispersity of number of arms is not very drastic but it does, however, change the relaxation pattern at intermediate times, leading to a faster relaxation of the crossbar compared to the relaxation predicted when using the average value of $q=2.5$.

More important is the effect of the the presence of asymmetric stars (half pom-pom's) in the melt. The picture is simplified by assuming that the asymmetric stars relax first. This is strictly not correct but the asymmetric star arms will somehow react faster than the arms emanating from a crossbar due to their more diluted surroundings. By applying the simple assumption that we have hierarchical relaxation in the different types of arm material, we can account for the dilution effect in a simple way. Rather than making the dilution continuous, we apply a step function which sets in at the longest relaxation time of the asymmetric star arms, i.e., when the innermost part of the star arm relaxes, and we regard the relaxation as a process in a pure star melt [Milner and McLeish (1998)].

Recently, Wagner et al. (2008) extended the MSF model [Wagner et al. (2005)] within the interchain pressure concept to include bidisperse (linear) polymers. It was demonstrated that the interchain pressure term introduced by Marrucci and Ianniruberto (2004), and the tube-diameter relaxation time concept following this, can be extended to understand the strain hardening of the long-chain component in the diluted regime. This presented constitutive approach is not in mathematical terms a constitutive model, as the time dependence of the tube-diameter relaxation time $\tau_{\text {dia }}$ was applied as a function of the constant elongational rate. During the stress relaxation, this rate is zero and potentially negative during reversed flow. However, the approach elucidates the importance of the dilution effect. To apply this approach to general flows we have introduced the time dependence, and not the extensional rate, directly into the extended tube-diameter relaxation time $\tau_{d i a}$ and the nonlinear spring coefficient $c$ as shown in Eq. (6). Compared to the model by Wagner et al. (2008), their equation (27), we assume that the stress originates from pom-pom molecules only and the presence of the stars is only considered as a dilution effect as discussed below. This is an acceptable assumption at low elongation rates where the asymmetric stars are considered relaxed. At high elongation rates the asymmetric stars in the melt is expected to contribute less to the steady viscosity than the pom-pom molecule. Based on the study on linear monodisperse polystyrenes by Bach et al. (2003) we estimate that the steady elongational viscosity to be approximately linear with the size of the molecule. The presence of the stars is then expected to lower the steady elongational viscosity (compared to the applied model) about $4 \%$ to $10 \%$ at high elongational rates, where the asymmetric stars are treated theoretically as pom-pom molecules. Notice the model from Wagner et al. (2008) did not include maximal extensibility.

The presence of the asymmetric stars in the melt give rise to a slight dilution effect on the maximal extensibility of $\lambda_{\max }\left(t-t^{\prime}\right)=5.07 / \sqrt{1-w\left(t-t^{\prime}\right)}$ and on the tube-diameter relaxation time of $\tau_{\text {dia }}\left(t-t^{\prime}\right)=\tau_{\text {dia, } 0} /\left(1-w\left(t-t^{\prime}\right)\right)^{2}$ for times above a characteristic relax- 
ation time of the asymmetric stars polystyrene in the melt. We have applied a $\tau_{\text {dia, } 0}$ $=120000 \mathrm{~s}$. The the longest relaxation time of the asymmetric stars is about $500 \mathrm{~s}$, as estimated from the Rouse relaxation time determined from the LVE data from Nielsen $e t$ al. (2006b). For simplicity the weight fraction is defined as $w\left(t-t^{\prime}\right)=0.2$ for $t-t^{\prime}$ $>500 \mathrm{~s}$ and $w\left(t-t^{\prime}\right)=0$ for $t-t^{\prime} \leqslant 500 \mathrm{~s}$, i.e., a step function rather than a continuous dilution in the entanglement spacing. Notice $\lambda_{\max }\left(t-t^{\prime}\right)$ is inserted into Eq. (7) to obtain a time dependent nonlinear spring coefficient $c$.

As seen in Fig. 2, the introduction of a dilution effect slightly reduced the deviation between data and the applied ideal monodisperse model. One exception is after the maximum appears in the transient elongational viscosity at large elongational rates. The appeared maximum in the transient elongational viscosity is commonly observed in broadly-distributed low-density polyethylene (LDPE) melts [Raible et al. (1979), Rasmussen et al. (2005)]. The applied model does not include this effect.

While the integral molecular stress function formulation based on the interchain pressure constitutes a simple explanation for the temporal development of the stretch evolution, we do not pursue the mechanism further here as it goes beyond the support of the presented data.

\section{CONCLUSIONS}

The filament stretching rheometer has been used to measure startup of uniaxial elongational flow followed by stress relaxation and reversed bi-axial flow for a NMMD branched polystyrene melt. The branched polystyrene melt is a NMMD multiarm $A_{\mathrm{q}}$ $-C-C-A_{\mathrm{q}}$ pom-pom polystyrene with a (number) average number of arms of approximately $q=2.5$.

The principle of time-strain separability fails completely to describe the data. The Doi-Edwards model with a stretch evolution equation is expected to be capable of predicting the stress relaxation measurements, in principle, whereas the Doi-Edwards model with any stretch evolution equation is not capable of capturing the level of recovery strain, in the reversed bi-axial flow.

The interchain pressure concept agrees with all the dynamics elongational measurements, showing the needs for time-dependent relaxation in the stretch contribution to the stress.

\section{ACKNOWLEDGMENTS}

The authors gratefully acknowledge financial support to the Graduate School of Polymer Science from the Danish Research Training Council and the Danish Technical Research Council to the Danish Polymer Centre and A. L. Skov, as well as the "Direktoer, dr. Techn. A.N. Neergaards og Hustrus Fond" foundation.

\section{References}

Archer, L. A., and Juliani, "Linear and nonlinear viscoelasticity of entangled multiarm (pom-pom) polymer liquids," Macromolecules 37, 1076-1088 (2004).

Bach, A., H. K. Rasmussen, P. Y. Longin, and O. Hassager, "Growth of non-axisymmetric disturbances of the free surface in the filament stretching rheometer: Experiments and simulation," J. Non-Newtonian Fluid Mech. 108, 163-186 (2002).

Bach, A., K. Almdal, H. K. Rasmussen, and O. Hassager, "Elongational viscosity of narrow molar mass 
distribution polystyrene," Macromolecules 36, 5174-5179 (2003a).

Bach, A., H. K. Rasmussen, and O. Hassager, "Extensional viscosity for polymer melts measured in the filament stretching rheometer," J. Rheol. 47, 429-441 (2003b).

Baumgaertel, M., A. Schausberger, and H. H. Winter, "The relaxation of polymers with linear flexible chains of uniform length,” Rheol. Acta 29, 400-408 (1990).

Bernstein, B., E. A. Kearsley, and L. J. Zapas, "Study of stress relaxation with finite strain,” Trans. Soc. Rheol. 7, 391-410 (1963).

Cohen, A., “A Pade approximant to the inverse Langevin function,” Rheol. Acta 30, 270-273 (1991).

de Gennes, P. G., "Reptation of a polymer chain in presence of fixed obstacles," J. Chem. Phys. 55, 572-579 (1971).

Doi, M., and S. F. Edwards, "Dynamics of concentrated polymer systems. 3. Constitutive equation," J. Chem. Soc., Faraday Trans. 2 74, 1818-1832 (1978).

Doi, M., and S. F. Edwards, "Dynamics of concentrated polymer systems. 4. Rheological properties," J. Chem. Soc., Faraday Trans. 2 75, 38-54 (1979).

Doi, M., and S. F. Edwards, The Theory of Polymer Dynamics (Clarendon, Oxford, UK, 1986).

Eriksson, T., and H. K. Rasmussen, "The effects of polymer melt rheology on the replication of surface microstructures in isothermal moulding," J. Non-Newtonian Fluid Mech. 127, 191-200 (2005).

Fang, J. N., M. Kroger, and H. C. Ottinger, "A thermodynamically admissible reptation model for fast flows of entangled polymers. II. Model predictions for shear and extensional flows," J. Rheol. 44, 1293-1317 (2000).

Fetters, L. J., D. J. Lohse, D. Richter, T. A. Witten, and A. Zirkel, "Connection between polymer molecular weight, density, chain dimensions, and melt viscosity properties," Macromolecules 27, 4639-4647 (1994).

Flory, P. J., "Theoretical predictions on configurations of polymer-chains in amorphous state," J. Macromol. Sci., Phys. 12, 1-11 (1976).

Hassager, O., M. I. Kolte, and M. Renardy, "Failure and nonfailure of fluid filaments in extension," J. NonNewtonian Fluid Mech. 76, 137-151 (1998).

Ianniruberto, G., and G. Marrucci, "A simple constitutive equation for entangled polymers with chain stretch," J. Rheol. 45, 1305-1318 (2001).

Jackson, J. K., and H. H. Winter, "Entanglement and flow behavior of bidisperse blends of polystyrene and polybutadiene," Macromolecules 28, 3146-3155 (1995).

Kaye, A., "College of Aeronautics," Cranheld, Note no. 134, 1962.

Knauss, D. M., and T. Z. Huang, "Star-block-linear-block-star triblock (pom-pom) polystyrene by convergent living anionic polymerization," Macromolecules 35, 2055-2062 (2002).

Kolte, M. I., H. K. Rasmussen, and O. Hassager, "Transient filament stretching rheometer. 2. Numerical simulation," Rheol. Acta 36, 285-302 (1997).

Likhtman, A. E., "Single-chain slip-link model of entangled polymers: Simultaneous description of neutron spin-echo, rheology, and diffusion," Macromolecules 38, 6128-6139 (2005).

Luap, C., C. Muller, T. Schweizer, and D. C. Venerus, "Simultaneous stress and birefringence measurements during uniaxial elongation of polystyrene melts with narrow molecular weight distribution," Rheol. Acta 45, 83-91 (2005).

Marrucci, G., and N. Grizzuti, "Fast flows of concentrated polymers-predictions of the tube model on chain stretching," Gazz. Chim. Ital. 118, 179-185 (1988).

Marrucci, G., and G. Ianniruberto, "Interchain pressure effect in extensional flows of entangled polymer melts," Macromolecules 37, 3934-3942 (2004).

Matallah, H., M. J. Banaai, K. S. Sujatha, and M. F. Webster, "Modelling filament stretching flows with strain-hardening models and sub-cell approximations," J. Non-Newtonian Fluid Mech. 134, 77-104 (2006).

Matallah, H., K. S. Sujatha, M. J. Banaai, and M. F. Webster, "Single and multi-mode modelling for filament stretching flows," J. Non-Newtonian Fluid Mech. 146, 92-113 (2007).

McLeish, T. C. B., J. Allgaier, D. K. Bick, G. Bishko, P. Biswas, R. Blackwell, B. Blottiere, N. Clarke, B. Gibbs, D. J. Groves, A. Hakiki, R. K. Heenan, J. M. Johnson, R. Kant, D. J. Read, and R. N. Young, "Dynamics of entangled H-polymers: Theory, rheology, and neutron-scattering," Macromolecules 32, 6734-6758 (1999). 
McLeish, T. C. B., and R. G. Larson, "Molecular constitutive equations for a class of branched polymers: The pom-pom polymer," J. Rheol. 42, 81-110 (1998).

Mead, D. W., R. G. Larson, and M. Doi, "A molecular theory for fast flows of entangled polymers," Macromolecules 31, 7895-7914 (1998).

Meissner, J., "Dehnungsverhalten von polyathylen-schmelzen,” Rheol. Acta 10, 230-242 (1971).

Meissner, J., and J. Hostettler, "A new elongational rheometer for polymer melts and other highly viscoelastic liquids," Rheol. Acta 33, 1-21 (1994).

Milner, S. T., and T. C. B. McLeish, "Arm-length dependence of stress relaxation in star polymer melts," Macromolecules 31, 7479-7482 (1998).

Münstedt, H., "New universal extensional rheometer for polymer melts. Measurements on a polystyrene sample," J. Rheol. 23, 421-436 (1979).

Nielsen, J. K., and H. K. Rasmussen, "Reversed extension flow," J. Non-Newtonian Fluid Mech. 155, 15-19 (2008).

Nielsen, J. K., H. K. Rasmussen, M. Denberg, K. Almdal, and O. Hassager, "Nonlinear branch-point dynamics of multiarm polystyrene," Macromolecules 39, 8844-8853 (2006a).

Nielsen, J. K., H. K. Rasmussen, O. Hassager, and G. H. McKinley, "Elongational viscosity of monodisperse and bidisperse polystyrene melts," J. Rheol. 50, 453-476 (2006b).

Nielsen, J. K., H. K. Rasmussen, and O. Hassager, "Stress relaxation of narrow molar mass distribution polystyrene following uni-axial extension,” J. Rheol. 52, 885-899 (2008).

Olley, P., and M. H. Wagner, "A modification of the convective constraint release mechanism in the molecular stress function model giving enhanced vortex growth," J. Non-Newtonian Fluid Mech. 135, 68-81 (2006).

Pearson, D. S., A. D. Kiss, L. J. Fetters, and M. Doi, "Flow-induced birefringence of concentrated polyisoprene solutions," J. Rheol. 33, 517-535 (1989).

Raible, T., A. Demarmels, and J. Meissner, "Stress and recovery maxima in LDPE melt elongation," Polym. Bull. (Berlin) 1, 397-402 (1979).

Rasmussen, H. K., and A. Bach, "On the bursting of linear polymer melts in inflation processes," Rheol. Acta 44, 435-445 (2005).

Rasmussen, H. K., and T. Eriksson, "Gas displacement of polymer melts in a cylinder: experiments and viscoelastic simulations," J. Non-Newtonian Fluid Mech. 143, 1-9 (2007).

Rasmussen, H. K., and O. Hassager, "Three-dimensional simulations of viscoelastic instability in polymeric filaments,” J. Non-Newtonian Fluid Mech. 82, 189-202 (1999).

Rasmussen, H. K., and O. Hassager, "The role of surface tension on the elastic decohesion of polymeric filaments," J. Rheol. 45, 527-537 (2001).

Rasmussen, H. K., P. Laillé, and K. Yu, "Large amplitude oscillatory elongation flow," Rheol. Acta 47, 97-103 (2008).

Rasmussen, H. K., J. K. Nielsen, A. Bach, and O. Hassager, "Viscosity overshoot in the start-up of uniaxial elongation of low density polyethylene melts," J. Rheol. 49, 369-381 (2005).

Rasmussen, H. K., and K. Yu, "On the burst of branched polymer melts during inflation," Rheol. Acta 47, 149-157 (2008).

Rolón-Garrido, V. H., M. H. Wagner, C. Luap, and T. Schweizer, "Modeling non-Gaussian extensibility effects in elongation of nearly monodisperse polystyrene melts," J. Rheol. 50, 327-340 (2006).

Rubio, P., and M. H. Wagner, "LDPE melt rheology and the pom-pom model," J. Non-Newtonian Fluid Mech. 92, 245-259 (2000).

Schieber, J. D., J. Neergaard, and S. Gupta, “A full-chain, temporary network model with sliplinks, chain-length fluctuations, chain connectivity and chain stretching," J. Rheol. 47, 213-233 (2003).

Sizaire, R., and V. Legat, "Element simulation of a filament stretching extensional rheometer," J. NonNewtonian Fluid Mech. 71, 89-107 (1997).

Sentmanat, M. L., "Miniature universal testing platform: From extensional melt rheology to solid-state deformation behavior," Rheol. Acta 43, 657-669 (2004).

Spiegelberg, S. H., D. C. Ables, and G. H. McKinley, "The role of end-effects on measurements of extensional viscosity in filament stretching rheometers,” J. Non-Newtonian Fluid Mech. 64, 229-267 (1996).

Sridhar, T., V. Tirtaatmadja, D. A. Nguyen, and R. K. Gupta, "Measurement of extensional viscosity of 
polymer-solutions,” J. Non-Newtonian Fluid Mech. 40, 271-280 (1991).

Sujatha, K. S., H. Matallah, M. J. Banaai, and M. F. Webster, "Computational predictions for viscoelastic filament stretching flows: ALE methods and free-surface techniques (CM and VOF)," J. Non-Newtonian Fluid Mech. 137, 81-102 (2006).

Szabo, P., "Transient filament stretching rheometer.1. Force balance analysis," Rheol. Acta 36, 277-284 (1997).

Szabo, P., and G. H. McKinley, "Filament stretching rheometer: Inertia compensation revisited," Rheol. Acta 42, 269-272 (2003).

Tanner, R. T., and K. Walters, Rheology: An Historical Perspective (Elsevier, Amstadam, 1998).

Wagner, M. H., "Constitutive analysis of uniaxial elongational flow data of a low-density polyethylene melt," J. Non-Newtonian Fluid Mech. 4, 39-55 (1978).

Wagner, M. H., P. Ehrecke, P. Hachmann, and J. Meissner, "A constitutive analysis of uniaxial, equibiaxial and planar extension of a commercial linear high-density polyethylene melt," J. Rheol. 42, 621-638 (1998).

Wagner, M. H., S. Kheirandish, and O. Hassager, "Quantitative prediction of transient and steady-state elongational viscosity of nearly monodisperse polystyrene melts," J. Rheol. 49, 1317-1327 (2005).

Wagner, M. H., V. H. Rolón-Garrido, J. K. Nielsen, H. K. Rasmussen, and O. Hassager, "A constitutive analysis of transient and steady-state elongational viscosities of bidisperse polystyrene blends," J. Rheol. 52, 67-86 (2008).

Wagner, M. H., and J. Schaeffer, "Nonlinear strain measures for general biaxial extension of polymer melts," J. Rheol. 36, 1-26 (1992).

Wang, Y., P. Boukany, S.-Q. Wang, and X. Wang, "Elastic breakup in uniaxial extension of entangled polymer melts," Phys. Rev. Lett. 99, 237801 (2007).

Webster, M. F., H. Matallah, K. S. Sujatha, and M. J. Banaai, "Numerical modelling of step-strain for stretched filaments," J. Non-Newtonian Fluid Mech. 151, 38-58 (2008).

Yao, M. W., G. H. McKinley, and B. Debbaut, "Extensional deformation, stress relaxation and necking failure of viscoelastic filaments," J. Non-Newtonian Fluid Mech. 70, 469-501 (1998).

Yao, M. W., S. H. Spiegelberg, and G. H. McKinley, "Dynamics of weakly strain-hardening fluids in filament stretching devices,” J. Non-Newtonian Fluid Mech. 89, 1-43 (2000).

Ye, X. N., and T. Sridhar, "Effects of the polydispersity on rheological properties of entangled polystyrene solutions," Macromolecules 38, 3442-3449 (2005). 